\title{
Positive Solutions for Nonlinear $q$-Fractional Difference Eigenvalue Problem with Nonlocal Conditions
}

\author{
Wafa Shammakh and Maryam Al-Yami \\ Sciences Faculty for Girls, King Abdulaziz University, Jeddah, Saudi Arabia \\ Correspondence should be addressed to Maryam Al-Yami; malyami@kau.edu.sa \\ Received 16 August 2015; Revised 5 November 2015; Accepted 26 November 2015 \\ Academic Editor: Svatoslav Staněk
}

Copyright (c) 2015 W. Shammakh and M. Al-Yami. This is an open access article distributed under the Creative Commons Attribution License, which permits unrestricted use, distribution, and reproduction in any medium, provided the original work is properly cited.

\begin{abstract}
The problem of positive solutions for nonlinear $q$-fractional difference eigenvalue problem with nonlocal boundary conditions is investigated. Based on the fixed point index theory in cones, sufficient existence of positive solutions conditions is derived for the problem.
\end{abstract}

\section{Introduction}

The fractional $q$-calculus is the $q$-extension of ordinary fractional calculus. It has been used by many researchers to adequately describe the evolution of a variety of engineering, economical, physical, and biological processes.

We consider a nonlinear $q$-fractional difference eigenvalue problem with nonlocal boundary conditions given by

$$
\begin{aligned}
{ }^{C} D_{q}^{\alpha} u(t)+\lambda g(t) f(t, u(t)) & =0, \\
& 0 \leq t \leq 1,0<q<1, \\
D_{q}^{k} u(0) & =0, \\
& u(0)=0,2 \leq k \leq n-1, \\
D_{q} u(1) & =\theta[u],
\end{aligned}
$$

where ${ }^{C} D_{q}^{\alpha}$ denote the fractional $q$-derivative of the Caputo type, $n-1<\alpha \leq n, n>2, \lambda>0$ is a parameter, and $\theta[u]$ is given by a Riemann-Stieltjes integral $\theta[u]=\int_{0}^{1} u(t) d_{q} A(t)$.

This type of $\mathrm{BC}$ includes, as particular cases, multipoint problems when $\theta[u]=\sum_{i=1}^{m-2} \alpha_{i} u\left(\zeta_{i}\right)$, (see [1]) and a continuously distributed case when $\theta[u]=\int_{0}^{1} \alpha(s) u(s) d_{q} s$ (see [24]).

More recently, many people pay attention to BVPs involving nonlinear $q$-difference equations [5-12].
In [13], Yuan and Yang dealt with some existence and uniqueness results for nonlinear boundary value problems for delayed $q$-fractional difference systems based on a contraction mapping principle and Krasnoselskii's fixed-point theorem.

In [14], Yang investigated the sufficient conditions for the existence and nonexistence positive solutions for BVP involving nonlinear $q$-fractional difference equations.

Ferreira [4] studied the existence of positive solutions to the nonlinear $q$-fractional BVPs by means of Krasnoselskii's fixed point theorem in cones.

In this paper, we obtain the results on the existence of one and two positive solutions by utilizing the results of Webb and Lan [15] involving comparison with the principle characteristic value of a related linear problem to the $q$ fractional case. We then use the theory worked out by Webb and Infante in [16-19] to study the general nonlocal BCs.

\section{Preliminaries}

In this section, we will present some definitions and lemmas that will be used in the proof of our main results.

Let $q \in(0,1)$ defined by [20]

$$
[a]_{q}=\frac{q^{a}-1}{q-1}=q^{a-1}+\cdots+1, \quad a \in \mathbb{R} .
$$


The $q$-analogue of the power function $(a-b)^{n}$ with $n \in \mathbb{N}$ is

$$
\begin{aligned}
(a-b)^{0} & =1 \\
(a-b)^{(n)} & =\prod_{k=0}^{n-1}\left(a-b q^{k}\right), \quad a, b \in \mathbb{R}, n \in \mathbb{N} .
\end{aligned}
$$

More generally, if $\alpha \in \mathbb{R}$, then

$$
(a-b)^{(\alpha)}=a^{\alpha} \prod_{i=0}^{\infty} \frac{\left(a-b q^{i}\right)}{\left(a-b q^{\alpha+i}\right)} .
$$

Note that if $b=0$ then $a^{(\alpha)}=a^{\alpha}$. The $q$-gamma function is defined by

$$
\begin{aligned}
& \Gamma_{q}(x)=\frac{(1-q)^{(x-1)}}{(1-q)^{x-1}} \\
& x \in \mathbb{R} \backslash\{0,-1,-2, \ldots\}, 0<q<1,
\end{aligned}
$$

and satisfies $\Gamma_{q}(x+1)=[x]_{q} \Gamma_{q}(x)$.

The $q$-derivative of a function $f(x)$ is here defined by

$$
D_{q} f(x)=\frac{d_{q} f(x)}{d_{q} x}=\frac{f(q x)-f(x)}{(q-1) x},
$$

and $q$-derivatives of higher order are defined by

$$
D_{q}^{n} f(x)= \begin{cases}f(x), & \text { if } n=0 \\ D_{q} D_{q}^{n-1} f(x) & \text { if } n \in \mathbb{N}\end{cases}
$$

The $q$-integral of a function $f$ defined in the interval $[0, b]$ is given by

$$
\begin{aligned}
\int_{0}^{x} f(t) d_{q} t=x(1-q) \sum_{n=0}^{\infty} f\left(x q^{n}\right) q^{n} & \\
& 0 \leq|q|<1, x \in[0, b] .
\end{aligned}
$$

If $a \in[0, b]$ and $f$ is defined in the interval $[0, b]$, its integral from $a$ to $b$ is defined by

$$
\int_{a}^{b} f(t) d_{q} t=\int_{0}^{b} f(t) d_{q} t-\int_{0}^{a} f(t) d_{q} t
$$

Similarly as done for derivatives, it can be defined an operator $I_{q}^{n}$; namely,

$$
\begin{aligned}
& \left(I_{q}^{0} f\right)(x)=f(x), \\
& \left(I_{q}^{n} f\right)(x)=I_{q}\left(I_{q}^{n-1} f\right)(x), \quad n \in \mathbb{N} .
\end{aligned}
$$

The fundamental theorem of calculus applies to these operators $I_{q}$ and $D_{q}$; that is,

$$
\left(D_{q} I_{q} f\right)(x)=f(x)
$$

and if $f$ is continuous at $x=0$, then

$$
\left(I_{q} D_{q} f\right)(x)=f(x)-f(0) .
$$

Basic properties of the two operators can be found in the book [20]. We now point out four formulas that will be used later:

$$
\begin{aligned}
& {[a(t-s)]^{(\alpha)}=a^{\alpha}(t-s)^{(\alpha)},} \\
& { }_{t} D_{q}(t-s)^{(\alpha)}=[\alpha]_{q}(t-s)^{(\alpha-1)}, \\
& { }_{s} D_{q}(t-s)^{(\alpha)}=-[\alpha]_{q}(t-q s)^{(\alpha-1)}, \\
& \left({ }_{x} D_{q} \int_{0}^{x} f(x, t) d_{q} t\right)(x) \\
& \quad=\int_{0}^{x} D_{x} f(x, t) d_{q} t+f(q x, x),
\end{aligned}
$$

where ${ }_{i} D_{q}$ denotes the $q$-derivative with respect to variable $i$ [21].

Remark 1 (see [21]). We note that if $\alpha>0$ and $a \leq b \leq t$, then $(t-a)^{(\alpha)} \geq(t-b)^{(\alpha)}$.

Definition 2 (see [22]). Let $\alpha \geq 0$ and let $f$ be a function defined on $[0,1]$. The fractional $q$-integral of the RiemannLiouville type is $\left({ }_{\mathrm{RL}} I_{q}^{0} f\right)(x)=f(x)$ and

$$
\begin{array}{r}
\left({ }_{\mathrm{RL}} I_{q}^{\alpha} f\right)(x)=\frac{1}{\Gamma_{q}(\alpha)} \int_{0}^{x}(x-q t)^{(\alpha-1)} f(t) d_{q} t, \\
\alpha \in \mathbb{R}^{+}, x \in[0,1] .
\end{array}
$$

Definition 3 (see [22]). The fractional $q$-derivative of the Riemann-Liouville type of order $\alpha \geq 0$ is defined by $\left({ }_{\mathrm{RL}} D_{q}^{0} f\right)(x)=f(x)$ and

$$
\left({ }_{\mathrm{RL}} D_{q}^{\alpha} f\right)(x)=\left(D_{q}^{[\alpha]} I_{q}^{[\alpha]-\alpha} f\right)(x), \quad \alpha>0,
$$

where $[\alpha]$ is the smallest integer greater than or equal to $\alpha$.

Definition 4 (see [22]). The fractional $q$-derivative of the Caputo type of order $\alpha \geq 0$ is defined by

$$
\left({ }_{C} D_{q}^{\alpha} f\right)(x)=\left(I_{q}^{[\alpha]-\alpha} D_{q}^{[\alpha]} f\right)(x), \quad \alpha>0 .
$$

Lemma 5 (see [22]). Let $\alpha, \beta \geq 0$ and let $f$ be a function defined on $[0,1]$. Then, the next formulas hold:

(1) $\left(I_{q}^{\beta} I_{q}^{\alpha} f\right)(x)=\left(I_{q}^{\alpha+\beta} f\right)(x)$,

(2) $\left({ }_{C} D_{q}^{\alpha} I_{q}^{\alpha} f\right)(x)=f(x)$. 
Lemma 6 (see [22]). Let $\alpha \in \mathbb{R}^{+} \backslash \mathbb{N}, \lambda \in(-1, \infty)$. Then, the next formulas hold:

(1) $I_{q}^{\alpha} x^{\lambda}=\frac{\Gamma_{q}(\lambda+1)}{\Gamma_{q}(\lambda+1+\alpha)} x^{\lambda+\alpha}$,
(2) ${ }_{R L} D_{q}^{\alpha} x^{\lambda}=\frac{\Gamma_{q}(\lambda+1)}{\Gamma_{q}(\lambda+\alpha+1)} x^{\lambda-\alpha}$,

(3)

$$
{ }_{C} D_{q}^{\alpha} x^{\lambda}= \begin{cases}0 & \text { if } \lambda \in \mathbb{N}_{0} ; \alpha>\lambda, \\ { }_{R L} D_{q}^{\alpha} x^{\lambda} & \text { if otherwise. }\end{cases}
$$

Theorem 7 (see [23]). Let $x>0$ and $\alpha \in \mathbb{R}^{+} \backslash \mathbb{N}$. Then, the following equality holds:

$$
\left(I_{q C}^{\alpha} D_{q}^{\alpha} f\right)(x)=f(x)-\sum_{k=0}^{[\alpha]-1} \frac{x^{k}}{\Gamma_{q}(k+1)}\left(D_{q}^{k} f\right)(0) .
$$

Lemma 8 (see [24]). Suppose $T: K \rightarrow K$ is a completely continuous operator and has no fixed points on $\partial K_{\rho} \cap K$. Then the following are true:

(i) If $\|T u\| \leq\|u\|$ for all $u \in \partial K_{\rho} \cap K$, then $i\left(T, K_{\rho} \cap\right.$ $K, K)=1$, where $i$ is the fixed point index on $K$.

(ii) If $\|T u\| \geq\|u\|$ for all $u \in \partial K_{\rho} \cap K$, then $i\left(T, K_{\rho} \cap\right.$ $K, K)=0$.

Lemma 9 (see [24]). Let $K$ be a cone in Banach space E. Suppose that $T: \bar{K}_{\rho} \rightarrow K$ is a completely continuous operator. There exists $u_{0} \in K \backslash\{0\}$ such that $u-T u \neq \mu u_{0}$ for any $u \in \partial K_{r}$ and $\mu \geq 0, i\left(T, K_{\rho}, K\right)=0$.

Lemma 10 (see [24]). Let $K$ be a cone in Banach space E. Suppose that $T: \bar{K}_{\rho} \rightarrow K$ is a completely continuous operator. If $T u \neq \mu u$ for any $u \in \partial K_{r}$ and $\mu \geq 1$, then $i\left(T, K_{\rho}, K\right)=1$.

Lemma 11. Let $y \in C[0,1]$ be a given function and $n-1<$ $\alpha \leq n$, then $u$ is a solution of BVP (1)-(2) if and only if $u$ is a solution of the integral equation

$$
u(t)=\gamma(t) \theta[u]+\int_{0}^{1} G_{0}(t, q s) y(s) d_{q} s,
$$

where

$$
\begin{aligned}
& \gamma(t)=t, \\
& G_{0}(t, q s) \\
& = \begin{cases}\frac{[\alpha-1]_{q} t(1-q s)^{(\alpha-2)}-(t-q s)^{(\alpha-1)}}{\Gamma_{q}(\alpha)}, & 0 \leq q s \leq t \leq 1, \\
\frac{[\alpha-1]_{q} t(1-q s)^{(\alpha-2)}}{\Gamma_{q}(\alpha)}, & 0 \leq t \leq q s \leq 1 .\end{cases}
\end{aligned}
$$

Proof. Assume that $u$ is a solution of BVP (1)-(2).

Applying Theorem 7, (1) can be reduced to an equivalent integral equation:

$$
\begin{aligned}
u(t)= & -\frac{1}{\Gamma_{q}(\alpha)} \int_{0}^{t}(t-q s)^{(\alpha-1)} y(s) d_{q} s+c_{0}+c_{1} t \\
& +c_{2} t^{2}+\cdots+c_{n-1} t^{n-1} .
\end{aligned}
$$

By (2), we obtain

$$
\begin{aligned}
& c_{0}=0, \\
& c_{2}=\cdots=c_{n-1}=0, \\
& c_{1}=\theta[u]+\frac{[\alpha-1]_{q}}{\Gamma_{q}(\alpha)} \int_{0}^{1}(1-q s)^{(\alpha-2)} y(s) d_{q} s .
\end{aligned}
$$

Therefore, we obtain

$$
\begin{aligned}
& u(t)=\gamma(t) \theta[u]+\frac{t[\alpha-1]_{q}}{\Gamma_{q}(\alpha)} \int_{0}^{1}(1-q s)^{(\alpha-2)} \\
& \cdot y(s) d_{q} s-\frac{1}{\Gamma_{q}(\alpha)} \int_{0}^{t}(t-q s)^{(\alpha-1)} y(s) d_{q} s \\
& =\gamma(t) \theta[u] \\
& +\int_{0}^{t}\left[\frac{[\alpha-1]_{q} t(1-q s)^{(\alpha-2)}-(t-q s)^{(\alpha-1)}}{\Gamma_{q}(\alpha)}\right] \\
& \cdot y(s) d_{q} s+\int_{t}^{1}\left[\frac{[\alpha-1]_{q} t(1-q s)^{(\alpha-2)}}{\Gamma_{q}(\alpha)}\right] \\
& \cdot y(s) d_{q} s=\gamma(t) \theta[u]+\int_{0}^{1} G_{0}(t, q s) y(s) d_{q} s .
\end{aligned}
$$

Conversely, if $u$ is a solution of the integral equation (20), using Lemmas 5 and 6, we have

$$
\begin{aligned}
{ }^{C} D_{q}^{\alpha} u(t) \\
={ }^{C} D_{q}^{\alpha} t \theta[u] \\
\quad+{ }^{C} D_{q}^{\alpha} t\left(\int_{0}^{1} \frac{[\alpha-1]_{q}(1-q s)^{(\alpha-2)}}{\Gamma_{q}(\alpha)} y(s) d_{q} s\right) \\
\quad-{ }^{C} D_{q}^{\alpha}\left(\int_{0}^{t} \frac{(t-q s)^{(\alpha-1)}}{\Gamma_{q}(\alpha)} y(s) d_{q} s\right) \\
=-{ }^{C} D_{q}^{\alpha} I_{q}^{\alpha} y(t)=-y(t) .
\end{aligned}
$$

A simple computation shows $u(0)=0, D_{q}^{k} u(0)=0,2 \leq k \leq$ $n-1, D_{q} u(1)=\theta[u]$.

Remark 12. $G_{0}(t, q s)$ is Green's function for the local BVP

$$
\begin{aligned}
&{ }^{C} D_{q}^{\alpha} u(t)+\lambda g(t) f(t, u(t))=0, \\
& t \in[0,1], n-1<\alpha \leq n, 0<q<1, \\
& D_{q}^{k} u(0)=0, \\
& u(0)=0,2 \leq k \leq n-1, \\
& D_{q} u(1)=0 .
\end{aligned}
$$


Lemma 13. Function $G_{0}(t, q s)$ defined in (20) satisfies the following conditions:

(H1) $G_{0}(t, q s) \geq 0$ is continuous and $G_{0}(t, s) \leq \Phi_{0}(q s)$ for all $0 \leq t, s \leq 1$;

(H2) $G_{0}(t, q s) \geq c_{0}(t) \Phi_{0}(q s)$ for all $0 \leq t, s \leq 1$, where

$$
\begin{aligned}
\Phi_{0}(q s) & =G_{0}(1, q s) \\
& =\frac{[\alpha-1]_{q}(1-q s)^{(\alpha-2)}-(1-q s)^{(\alpha-1)}}{\Gamma_{q}(\alpha)},
\end{aligned}
$$

$$
c_{0}(t)=t^{\alpha-1}
$$

Proof. It is obvious that $G_{0}(t, q s)$ is nonnegative and continuous.

(H1) For $0 \leq q s \leq t \leq 1$,

$$
\begin{aligned}
& G_{0}(t, q s)=\frac{1}{\Gamma_{q}(\alpha)}\left[[\alpha-1]_{q} t(1-q s)^{(\alpha-2)}\right. \\
& \left.\quad-(t-q s)^{(\alpha-1)}\right]=\frac{1}{\Gamma_{q}(\alpha)}\left[[\alpha-1]_{q} t(1-q s)^{(\alpha-2)}\right. \\
& \left.\quad-t^{\alpha-1}\left(1-q \frac{s}{t}\right)^{(\alpha-1)}\right] \\
& \quad \geq \frac{1}{\Gamma_{q}(\alpha)}\left[[\alpha-1]_{q} t^{\alpha-1}(1-q s)^{(\alpha-2)}\right. \\
& \left.-t^{\alpha-1}(1-q s)^{(\alpha-1)}\right] \\
& \quad=\frac{t^{\alpha-1}}{\Gamma_{q}(\alpha)}\left[[\alpha-1]_{q}(1-q s)^{(\alpha-2)}-(1-q s)^{(\alpha-1)}\right] \\
& \geq 0
\end{aligned}
$$

and for $0 \leq t \leq q s \leq 1$,

$$
G_{0}(t, q s)=\frac{[\alpha-1]_{q} t(1-q s)^{(\alpha-2)}}{\Gamma_{q}(\alpha)},
$$

and it is clear that $G_{0}(t, q s) \geq 0$ and $G_{0}(0, q s)=0$. Therefore $G_{0}(t, q s) \geq 0$.

For fixed $s \in[0,1]$ and $t \geq q s$ we have

$$
\begin{aligned}
{ }_{t} D_{q} G_{0}(t, q s) \\
=\frac{[\alpha-1]_{q}(1-q s)^{(\alpha-2)}-[\alpha-1]_{q}(t-q s)^{(\alpha-2)}}{\Gamma_{q}(\alpha)} \\
=\frac{(1-q s)^{(\alpha-2)}-(t-q s)^{(\alpha-2)}}{\Gamma_{q}(\alpha-1)} \geq 0
\end{aligned}
$$

that is, $G_{0}(t, q s)$ is an increasing function of $t$. Obviously, $G_{0}(t, q s), t \leq q s$, is increasing in $t$; therefore $G_{0}(t, q s)$ is an increasing function of $t$ for fixed $s \in[0,1]$.

Thus, (H1) holds.
(H2) Suppose now that $t \geq q$ :

$$
\begin{aligned}
& \frac{G_{0}(t, q s)}{\Phi_{0}(q s)}=\frac{[\alpha-1]_{q} t(1-q s)^{(\alpha-2)}-(t-q s)^{(\alpha-1)}}{[\alpha-1]_{q}(1-q s)^{(\alpha-2)}-(1-q s)^{(\alpha-1)}} \\
& \geq \frac{t^{\alpha-1}\left[[\alpha-1]_{q}(1-q s)^{(\alpha-2)}-(1-q s)^{(\alpha-1)}\right]}{[\alpha-1]_{q}(1-q s)^{(\alpha-2)}-(1-q s)^{(\alpha-1)}} \\
& =t^{\alpha-1} .
\end{aligned}
$$

On the other hand, if $t \leq q s$, then we have

$$
\begin{aligned}
& \frac{G_{0}(t, q s)}{\Phi_{0}(q s)}=\frac{[\alpha-1]_{q} t(1-q s)^{(\alpha-2)}}{[\alpha-1]_{q}(1-q s)^{(\alpha-2)}-(1-q s)^{(\alpha-1)}} \\
& \geq \frac{[\alpha-1]_{q} t^{\alpha-1}(1-q s)^{(\alpha-2)}-t^{\alpha-1}(1-q s)^{(\alpha-1)}}{[\alpha-1]_{q}(1-q s)^{(\alpha-2)}-(1-q s)^{(\alpha-1)}} \\
& =t^{\alpha-1},
\end{aligned}
$$

and this finished the proof of (H2).

Defining $\mathscr{G}_{A}(q s)=\int_{0}^{1} G_{0}(t, q s) d_{q} A(t)$, Green's function for nonlocal BVP (1)-(2) is given by

$$
G(t, q s)=\frac{\gamma(t)}{[1-\theta[\gamma]]} \mathscr{G}_{A}(q s)+G_{0}(t, q s) .
$$

Throughout the paper we assume the following:

(H3) $\mathrm{A}$ is a function of bounded variation, and $\mathscr{G}_{A}(q s)=$ $\int_{0}^{1} G_{0}(t, q s) d_{q} A(t)$ satisfies $\mathscr{G}_{A}(q s) \geq 0$ for almost every $s \in$ $[0,1]$. Note that $\mathscr{G}_{A}(q s)$ exists for almost every $s$ by $(\mathbf{H 1})$.

(H4) The functions $g$, $\Phi$ satisfy $g \geq 0$ almost everywhere, $g \Phi \in L^{1}[0,1]$, and

$$
\int_{a}^{b} \Phi(q s) g(s) d_{q} s>0 .
$$

(H5) $f:[0,1] \times[0, \infty) \rightarrow[0, \infty)$ satisfies Caratheodory conditions; that is, $f(\cdot, u)$ is measurable for each fixed $u \in$ $[0, \infty)$ and $f(t, \cdot)$ is continuous for almost every $t \in[0,1]$, and for each $r>0$, there exists $\phi_{r} \in L^{\infty}[0,1]$ such that $0 \leq$ $f(t, u) \leq \phi_{r}$ for all $u \in[0, r]$ and almost all $t \in[0,1]$.

(H6) One has the following: $\gamma \in C[0,1], \gamma(t) \geq 0,0 \leq$ $\theta[\gamma]<1$.

Lemma 14. If $G_{0}$ satisfies (H1), (H2), then $G$ satisfies (H1), (H2) for a function $\Phi$, the same interval $[a, b]$, and the same constant $c$, where $\Phi$ satisfies (H4) and $c=\min \left\{c_{0}(t): t \in\right.$ $[a, b]\}$.

Proof. We have

$$
\begin{aligned}
G(t, q s) & =\frac{\gamma(t)}{[1-\theta[\gamma]]} \mathscr{G}_{A}(q s)+G_{0}(t, q s) \\
& \leq \frac{\|\gamma\|}{[1-\theta[\gamma]]} \mathscr{G}_{A}(q s)+\Phi_{0}(q s)=: \Phi(q s),
\end{aligned}
$$


and for $t \in[a, b]$

$$
G(t, q s) \geq \frac{c\|\gamma\|}{[1-\theta[\gamma]]} \mathscr{G}_{A}(q s)+c \Phi_{0}(q s)=c \Phi(q s) .
$$

Note that $g \Phi \in L^{\infty}$ because $A$ has finite variation and $\mathscr{G}_{A}(q s) \leq \Phi(q s) \operatorname{var}(A)$.

Thus, Green's function $G(t, q s)$ satisfies $(\mathrm{H} 1),(\mathrm{H} 2)$ for a function $\Phi$ and the constant $c$.

\section{Main Result}

Set $E=C[0,1]$ as a Banach space with the norm $\|u\|=$ $\sup _{t \in[0,1]}|u(t)|$. Let $P=\{u \in E: u \geq 0\}$ denote the standard cone of nonnegative functions. Define

$$
K=\left\{u \in P, \min _{a \leq t \leq b} u(t) \geq c\|u\|\right\},
$$

where $[a, b]$ is some subset of $[0,1]$.

Note that $\gamma \in K$ so $K \neq\{0\}$. For any $0<r<R<+\infty$, let $K_{r}=\{u \in K:\|u\|<r\}, \partial K_{r}=\{u \in K:\|u\|=r\}$, $\bar{K}_{r}=\{u \in K:\|u\| \leq r\}, \bar{K}_{R} \backslash K_{r}=\{u \in K: r \leq\|u\| \leq R\}$, and $V_{r}=\left\{u \in K: \min _{t \in[a, b]} u(t)<r\right\}$ and $V_{r}$ is bounded.

Define a nonlinear operator $T: P \rightarrow K$ and a linear operator $L: P \rightarrow K$ by

$$
\begin{aligned}
& T u(t)=\lambda \int_{0}^{1} G(t, q s) g(s) f(s, u(s)) d_{q} s, \\
& \operatorname{Lu}(t):=\int_{0}^{1} G(t, q s) g(s) u(s) d_{q} s .
\end{aligned}
$$

Lemma 15 (see [18]). Under hypotheses (H1)-(H6) the maps $T: P \rightarrow$ E defined in (38) are compact.

Theorem 16. Under hypotheses $(\mathbf{H 1})-(\mathbf{H 6})$ the maps are $T$ : $P \rightarrow K$.

Proof. For $u \in P$ and $t \in[0,1]$ we have

$$
T u(t) \leq \lambda \int_{0}^{1} \Phi(q s) g(s) f(s, u(s)) d_{q} s .
$$

Hence,

$$
\|T u\| \leq \lambda \int_{0}^{1} \Phi(q s) g(s) f(s, u(s)) d_{q} s .
$$

Also, for $t \in[a, b]$, we have

$$
T u(t) \geq c \lambda \int_{0}^{1} \Phi(q s) g(s) f(s, u(s)) d_{q} s \geq c\|T u\| .
$$

Similar to the proofs of Lemma 15 and Theorem $16, L u(t)$ is compact and maps $P$ into $K$.

We will use the Krein-Rutman theorem. We recall that $\lambda$ is an eigenvalue of $L$ with corresponding eigenfunction $\phi$ if $\phi \neq 0$ and $\lambda \phi=L \phi$. The reciprocals of eigenvalues are called characteristic values of $L$. The radius of the spectrum of $L$, denoted by $r(L)$, is given by the well-known spectral radius formula $r(L)=\lim _{n \rightarrow \infty}\left\|L^{n}\right\|^{1 / n}$.
Theorem 17 (see [15]). Let $K$ be a total cone in a real Banach space $E$ and let $\widehat{L}: E \rightarrow E$ be a compact linear operator with $\widehat{L}(K) \subseteq K$. If $r(\widehat{L})>0$ then there is $\phi_{1} \in K \backslash\{0\}$ such that $\widehat{L} \phi_{1}=r(\widehat{L}) \phi_{1}$.

Thus $\lambda_{1}:=r(\widehat{L})$ is an eigenvalue of $\widehat{L}$, the largest possible real eigenvalue, and $\mu_{1}=1 / \lambda_{1}$ is the smallest positive characteristic value.

Lemma 18 (see [15]). Assume that (H1)-(H3) hold and let L be as defined in (39). Then $r(L)>0$.

Theorem 19 (see [15]). When (H1)-(H3) hold, $r(L)$ is an eigenvalue of $L$ with eigenfunction $\phi_{1}$ in $K$.

Theorem 20 (see [15]). Let $\mu_{1}=1 / r(L)$ and $\phi_{1}(t)$ be a corresponding eigenfunction in P of norm 1 . Then $m \leq \mu_{1} \leq M$, where

$$
\begin{gathered}
m=\left(\sup _{t \in[0,1]} \int_{0}^{1} G(t, q s) g(s) d_{q} s\right)^{-1}, \\
M=\left(\inf _{t \in[a, b]} \int_{a}^{b} G(t, q s) g(s) d_{q} s\right)^{-1} .
\end{gathered}
$$

If $g(t)>0$ for $t \in[0,1]$ and $G(t, q s)>0$ for $t, s \in[0,1]$, the first inequality is strict unless $\phi_{1}(t)$ is constant for $t \in[0,1]$. If $g(t) \phi(t)>0$ for $t \in[a, b]$, the second inequality is strict unless $\phi_{1}(t)$ is constant for $t \in[a, b]$.

Proof (for the local BVP (1)-(2) if $g(t) \equiv 1)$. We now compute the constant $m$ and the optimal value of $M(a, b)$; that is, we determine $a, b$ so that $M(a, b)$ is minimal.

For $q s \leq t$, we have by direct integration

$$
\begin{aligned}
& \int_{0}^{t} G_{0}(t, q s) d_{q} s \\
& \quad=\int_{0}^{t}\left[\frac{[\alpha-1]_{q} t(1-q s)^{(\alpha-2)}-(t-q s)^{(\alpha-1)}}{\Gamma_{q}(\alpha)}\right] d_{q} s \\
& \quad=\frac{t-t(1-t)^{(\alpha-1)}}{\Gamma_{q}(\alpha)}-\frac{t^{\alpha}}{[\alpha]_{q} \Gamma_{q}(\alpha)} .
\end{aligned}
$$

For $q s \geq t$

$$
\begin{aligned}
\int_{t}^{1} G_{0}(t, q s) d_{q} s & =\int_{t}^{1} \frac{[\alpha-1]_{q} t(1-q s)^{(\alpha-2)}}{\Gamma_{q}(\alpha)} d_{q} s \\
& =\frac{t(1-t)^{(\alpha-1)}}{\Gamma_{q}(\alpha)} .
\end{aligned}
$$

Then we have

$$
\int_{0}^{1} G_{0}(t, q s) d_{q} s=\frac{t}{\Gamma_{q}(\alpha)}-\frac{t^{\alpha}}{[\alpha]_{q} \Gamma_{q}(\alpha)}
$$


And the maximum of this expression occurs when $t=1$; hence

$$
\begin{aligned}
& \sup _{t \in[0,1]} \int_{0}^{1} G_{0}(t, q s) d_{q} s=\frac{1}{\Gamma_{q}(\alpha)}-\frac{1}{[\alpha]_{q} \Gamma_{q}(\alpha)} \\
& \quad=\frac{[\alpha]_{q}-1}{[\alpha]_{q} \Gamma_{q}(\alpha)} .
\end{aligned}
$$

Then $m=[\alpha]_{q} \Gamma_{q}(\alpha) /\left([\alpha]_{q}-1\right)$.

For $a<b$, we have by direct integration

$$
\begin{aligned}
\int_{a}^{t} G_{0}(t, q s) d_{q} s= & -\frac{t(1-t)^{(\alpha-1)}}{\Gamma_{q}(\alpha)}+\frac{t(1-a)^{(\alpha-1)}}{\Gamma_{q}(\alpha)} \\
& -\frac{(t-a)^{(\alpha)}}{[\alpha]_{q} \Gamma_{q}(\alpha)} \\
\int_{t}^{b} G_{0}(t, s) d s= & -\frac{t(1-b)^{(\alpha-1)}}{\Gamma_{q}(\alpha)}+\frac{t(1-t)^{(\alpha-1)}}{\Gamma_{q}(\alpha)} .
\end{aligned}
$$

Then

$$
\begin{aligned}
\int_{a}^{b} G_{0}(t, q s) d_{q} s= & \frac{t(1-a)^{(\alpha-1)}}{\Gamma_{q}(\alpha)}-\frac{(t-a)^{(\alpha)}}{[\alpha]_{q} \Gamma_{q}(\alpha)} \\
& -\frac{t(1-b)^{(\alpha-1)}}{\Gamma_{q}(\alpha)} \\
= & \frac{t\left[(1-a)^{(\alpha-1)}-(1-b)^{(\alpha-1)}\right]}{\Gamma_{q}(\alpha)} \\
& -\frac{(t-a)^{(\alpha)}}{[\alpha]_{q} \Gamma_{q}(\alpha)}=R(t, a, b), \\
D_{q} R(t, a, b)= & \frac{\left[(1-a)^{(\alpha-1)}-(1-b)^{(\alpha-1)}\right]}{\Gamma_{q}(\alpha)} \\
& -\frac{(t-a)^{(\alpha-1)}}{\Gamma_{q}(\alpha)} .
\end{aligned}
$$

The sign of derivative ${ }_{t} D_{q} R$ shows that this is an increasing function of $t$ so the minimum occurs at $t=a$. Let

$$
R(a, b)=\frac{a}{\Gamma_{q}(\alpha)}\left[(1-a)^{(\alpha-1)}-(1-b)^{(\alpha-1)}\right] .
$$

The minimal value of $M(a, b)$ corresponds to the maximal value of $R(a, b)$. Consider

$$
{ }_{b} D_{q} R(a, b)=\frac{a[\alpha-1]_{q}(1-q b)^{(\alpha-2)}}{\Gamma_{q}(\alpha)}>0 .
$$

The quantity $R(a, b)$ is an increasing function of $b$ so its maximum occurs when $b=1$. Let

$$
R(a)=\frac{a(1-a)^{(\alpha-1)}}{\Gamma_{q}(\alpha)} .
$$

Then the maximum of $R(a)$ occurs when $a=1 /\left(1+[\alpha-1]_{q}\right)$. Consider

$$
\min _{t \in[a, b]} \int_{a}^{b} G_{0}(t, q s) d_{q} s=R\left(\frac{1}{1+[\alpha-1]_{q}}, 1\right) .
$$

Hence the minimal value of $M(a, b)$ is

$$
M\left(\frac{1}{1+[\alpha-1]_{q}}, 1\right)=\left(R\left(\frac{1}{1+[\alpha-1]_{q}}, 1\right)\right)^{-1}
$$

\section{The Existence of at Least One Positive Solution}

For convenience, we introduce the following notations:

$$
\begin{aligned}
\bar{f}(u) & :=\sup _{t \in[0,1]} f(t, u), \\
\underline{f}(u) & :=\inf _{t \in[0,1]} f(t, u) ; \\
f^{0} & :=\limsup _{u \rightarrow 0^{+}} \frac{\bar{f}(u)}{u}, \\
f_{0} & :=\liminf _{u \rightarrow 0^{+}} \frac{f(u)}{u} ; \\
f^{\infty} & :=\limsup _{u \rightarrow \infty} \frac{\bar{f}(u)}{u}, \\
f_{\infty} & :=\liminf _{u \rightarrow \infty} \frac{f}{u}, \\
f^{0, r} & :=\sup _{\{0 \leq t \leq 1,0 \leq u \leq r\}} \frac{f(t, u)}{r}, \\
f_{r, r / c} & :=\inf _{\{a \leq t \leq b, r \leq u \leq r / c\}} \frac{f(t, u)}{r} .
\end{aligned}
$$

Under hypotheses (H1)-(H4) let $\widetilde{L}$ be defined by

$$
\widetilde{L} u(t)=\int_{a}^{b} G(t, q s) g(s) u(s) d_{q} s
$$

Then $\widetilde{L}$ is a compact linear operator and $\widetilde{L}(P) \subseteq K$.

Hence $r(\widetilde{L})$ is an eigenvalue of $\widetilde{L}$ with an eigenfunction $\widetilde{\phi}_{1}$ in $K$. Let $\tilde{\mu}_{1}:=1 / r(\widetilde{L})$. Note that $\tilde{\mu}_{1} \geq \mu_{1}$; hence the condition in the following theorem is more stringent compared with the case if $r(L)$ could be used.

Theorem 21. Assume that

$$
\begin{aligned}
& \text { (A1) } 0 \leq \lambda f^{0}<\mu_{1}, \\
& \text { (A2) } \tilde{\mu}_{1}<\lambda f_{\infty} \leq \infty .
\end{aligned}
$$

Then (1)-(2) had at least one positive solution. 
Proof. Let $\varepsilon>0$ be such that $f^{0} \leq(1 / \lambda)\left(\mu_{1}-\varepsilon\right)$. Then there exists $\rho_{0}>0$ such that

$$
f(t, u) \leq \frac{1}{\lambda}\left(\mu_{1}-\varepsilon\right) u,
$$

$\forall u \in\left[0, \rho_{0}\right]$ and almost all $t \in[0,1]$.

Let $\rho \in\left(0, \rho_{0}\right]$. We prove that

$$
T u \neq \beta u \quad \text { for } u \in \partial K_{\rho}, \beta \geq 1,
$$

which implies the result. In fact, if (58) does not hold, then there exist $u \in \partial K_{\rho}$ and $\beta \geq 1$ such that $T u=\beta u$.

This implies

$$
\begin{aligned}
\beta u(t) & =\lambda \int_{0}^{1} G(t, q s) g(s) f(s, u(s)) d_{q} s \\
& \leq\left(\mu_{1}-\varepsilon\right) \int_{0}^{1} G(t, q s) g(s) u(s) d_{q} s \\
& =\left(\mu_{1}-\varepsilon\right) L u(t) .
\end{aligned}
$$

Thus, we have shown $u(t) \leq\left(\mu_{1}-\varepsilon\right) L u(t)$. This gives

$$
\begin{aligned}
u(t) & \leq\left(\mu_{1}-\varepsilon\right) L\left[\left(\mu_{1}-\varepsilon\right) L u(t)\right] \\
& =\left(\mu_{1}-\varepsilon\right)^{2} L^{2} u(t) .
\end{aligned}
$$

And by iterating

$$
u(t) \leq\left(\mu_{1}-\varepsilon\right)^{n} L^{n} u(t) \quad \text { for } n \in N .
$$

Therefore

$$
\begin{aligned}
\|u\| & \leq\left(\mu_{1}-\varepsilon\right)^{n}\left\|L^{n}\right\|\|u\|, \\
1 & \leq\left(\mu_{1}-\varepsilon\right)^{n}\left\|L^{n}\right\|,
\end{aligned}
$$

and we have

$$
1 \leq\left(\mu_{1}-\varepsilon\right) \lim _{n \rightarrow+\infty}\left\|L^{n}\right\|^{1 / n}=\left(\mu_{1}-\varepsilon\right) \frac{1}{\mu_{1}}<1,
$$

a contradiction. It follows that

$$
i_{k}\left(T, K_{\rho}\right)=1, \quad \text { for each } \rho \in\left(0, \rho_{0}\right] .
$$

Let $\rho_{1}>0, \rho_{1}>\rho$ be chosen so that $f(t, u)>\left(\widetilde{\mu}_{1} / \lambda\right) u$ for all $u \geq c \rho_{1}, c$ as in (H2), and almost all $t \in[0,1]$.

We claim that $u \neq T u+\beta \widetilde{\phi}_{1}$ for all $\beta>0$ and $u \in \partial K_{\rho^{*}}$ when $\rho^{*}>\rho_{1}$. Note that $u \in K$ with $\|u\|=\rho^{*} \geq \rho_{1}$.

We have $u(t) \geq c \rho_{1}$ for all $t \in[a, b]$.

Now, if our claim is false, then we have

$$
u(t)=\lambda \int_{0}^{1} G(t, q s) g(s) f(s, u(s)) d_{q} s+\beta \widetilde{\phi}_{1}(t) .
$$

Therefore,

$$
\begin{aligned}
u(t) & \geq \widetilde{\mu}_{1} \int_{a}^{b} G(t, q s) g(s) u(s) d_{q} s+\beta \widetilde{\phi}_{1}(t) \\
& =\widetilde{\mu}_{1} \widetilde{L} u(t)+\beta \widetilde{\phi}_{1}(t) .
\end{aligned}
$$

From (66) we firstly deduce that $u(t) \geq \beta \widetilde{\phi}_{1}(t)$ on $[0,1]$.
Then we have

$$
\widetilde{\mu}_{1} \widetilde{L} u(t) \geq \widetilde{\mu}_{1} \widetilde{L}\left(\beta \widetilde{\phi}_{1}(t)\right)=\beta \widetilde{\phi}_{1}(t) .
$$

Inserting this into (66) we obtain $u(t) \geq 2 \beta \widetilde{\phi}_{1}(t)$ for $t \in[0,1]$. Repeating this process gives

$$
u(t) \geq n \beta \widetilde{\phi}_{1}(t) \quad \text { for } t \in[0,1], n \in N .
$$

Since $\widetilde{\phi}_{1}(t)$ is strictly positive on $[0,1]$ this is a contradiction; then

$$
i_{K}\left(T, K_{\rho^{*}}\right)=0, \quad \text { for } u \in \partial K_{\rho^{*}} .
$$

By (64) and (69), one has

$$
i_{K}\left(T, K_{\rho^{*}} \backslash \bar{K}_{\rho}\right)=i_{K}\left(T, K_{\rho^{*}}\right)-i_{K}\left(T, K_{\rho}\right)=-1 .
$$

Therefore, $T$ has at least one fixed point $u_{0} \in K_{\rho^{*}} \backslash \bar{K}_{\rho}$, and $u_{0}$ is a positive solution of BVP (1)-(2).

Theorem 22. Assume that

$$
\begin{aligned}
& \text { (A3) } \mu_{1}<\lambda f_{0} \leq \infty, \\
& \text { (A4) } 0 \leq \lambda f^{\infty}<\mu_{1} .
\end{aligned}
$$

Then (1)-(2) had at least one positive solution.

Proof. Let $\varepsilon>0$ satisfy $f_{0}>(1 / \lambda)\left(\mu_{1}+\varepsilon\right)$. Then there exists $R_{1}>0$ such that

$$
f(t, u) \geq \frac{1}{\lambda}\left(\mu_{1}+\varepsilon\right) u, \quad \forall t \in[0,1], u \in\left[0, R_{1}\right] .
$$

For any $u \in \partial K_{R_{1}}$ we have by (71) that

$$
\begin{aligned}
T u(t) & =\lambda \int_{0}^{1} G(t, q s) g(s) f(s, u(s)) d_{q} s \\
& \geq\left(\mu_{1}+\varepsilon\right) \int_{0}^{1} G(t, q s) g(s) u(s) d_{q} s \\
& \geq \mu_{1} L u(t), \quad \forall t \in[0,1] .
\end{aligned}
$$

Let $\widetilde{u}_{1}$ be the positive eigenfunction of $L$ corresponding to $\mu_{1}$; that is, $\widetilde{u}_{1}=\mu_{1} L \widetilde{u}_{1}$. We may suppose that $T$ has no fixed point on $\partial K_{R_{1}}$; otherwise, the proof is finished. In the following we will show that

$$
u-T u \neq \beta \widetilde{u}_{1}, \quad \forall u \in \partial K_{R_{1}}, \beta \geq 0 .
$$

If (73) is not true, then there is $\widetilde{u}_{0} \in \partial K_{R_{1}}$ and $\beta_{0} \geq 0$ such that $\tilde{u}_{0}-T \tilde{u}_{0}=\beta_{0} \tilde{u}_{1}$. It is clear that $\beta_{0}>0$ and $\tilde{u}_{0}=T \tilde{u}_{0}+\beta_{0} \tilde{u}_{1} \geq$ $\beta_{0} \tilde{u}_{1}$.

Set

$$
\beta^{*}=\sup \left\{\beta: \widetilde{u}_{0} \geq \beta \widetilde{u}_{1}\right\} .
$$

Obviously, $\beta^{*} \geq \beta_{0}>0$. It follows from $L(P) \subset P$ that

$$
\mu_{1} L \widetilde{u}_{0} \geq \mu_{1} L \beta^{*} \widetilde{u}_{1}=\beta^{*} \mu_{1} L \widetilde{u}_{1}=\beta^{*} \widetilde{u}_{1},
$$


and using this and (72), we have

$$
\tilde{u}_{0}=T \tilde{u}_{0}+\beta_{0} \tilde{u}_{1} \geq \mu_{1} L \tilde{u}_{0}+\beta_{0} \tilde{u}_{1} \geq \beta^{*} \tilde{u}_{1}+\beta_{0} \tilde{u}_{1},
$$

which contradicts (74). Thus, (73) holds.

By Lemma 9, we have

$$
i_{K}\left(T, K_{R_{1}}\right)=0 .
$$

On the other hand, let $\varepsilon>0$ satisfy $f^{\infty}<(1 / \lambda)\left(\mu_{1}-\varepsilon\right)$. Then there exists $R_{2}>R_{1}$ such that

$$
f(t, u) \leq \frac{1}{\lambda}\left(\mu_{1}-\varepsilon\right) u, \quad \forall t \in[0,1], u \geq R_{2} .
$$

By (H5) there exists an $L^{\infty}$ function $\varphi_{1}$ such that

$$
f(t, u) \leq \frac{1}{\lambda} \varphi_{1}(t), \quad \forall u \in\left[0, R_{2}\right], t \in[0,1] .
$$

Hence, we have

$$
f(t, u) \leq \frac{1}{\lambda}\left[\left(\mu_{1}-\varepsilon\right) u+\varphi_{1}(t)\right],
$$

$$
\forall u \in R^{+}, t \in[0,1] \text {. }
$$

Since $1 / \mu_{1}$ is the radius of the spectrum of $L,\left(I /\left(\mu_{1}-\varepsilon\right)-L\right)^{-1}$ exists.

Let

$$
\begin{aligned}
C & =\int_{0}^{1} \varphi_{1}(s) \Phi(s) g(s) d_{q} s \\
R_{0} & =\left(\frac{I}{\left(\mu_{1}-\varepsilon\right)}-L\right)^{-1}\left(\frac{c}{\left(\mu_{1}-\varepsilon\right)}\right) .
\end{aligned}
$$

We prove that, for each $R>R_{0}$,

$$
T u \neq \beta u, \quad \forall u \in \partial K_{R}, \beta \geq 1 .
$$

In fact, if not, there exist $u \in \partial K_{R}$ and $\beta \geq 1$ such that $T u=$ $\beta u$.

This together with (80) implies

$$
\begin{aligned}
u(t) \leq & \int_{0}^{1} G(t, q s) g(s)\left(\left(\mu_{1}-\varepsilon\right) u(s)+\varphi_{1}(s)\right) d_{q} s \\
= & \left(\mu_{1}-\varepsilon\right) \int_{0}^{1} G(t, q s) g(s) u(s) d_{q} s \\
& +\int_{0}^{1} G(t, q s) g(s) \varphi_{1}(s) d_{q} s \\
\leq & \left(\mu_{1}-\varepsilon\right) \operatorname{Lu}(t)+C .
\end{aligned}
$$

This implies

$$
\begin{aligned}
\left(\frac{I}{\mu_{1}-\varepsilon}-L\right) u(t) & \leq \frac{C}{\mu_{1}-\varepsilon}, \\
u(t) & \leq\left(\frac{I}{\mu_{1}-\varepsilon}-L\right)^{-1}\left(\frac{C}{\mu_{1}-\varepsilon}\right) \\
& =R_{0} .
\end{aligned}
$$

Therefore, we have $\|u\| \leq R_{0}<R$, a contradiction. Taking $R>R_{2}$, it follows from (74) and properties of index that

$$
i_{K}\left(T, K_{R}\right)=1, \quad \forall R>R_{0} .
$$

Now (77) and (85) combined imply

$$
i_{K}\left(T, K_{R} \backslash \bar{K}_{R_{1}}\right)=i_{K}\left(T, K_{R}\right)-i_{K}\left(T, \bar{K}_{R_{1}}\right)=1 .
$$

Therefore, $T$ has at least one fixed point $u_{0} \in K_{R} / \bar{K}_{R_{1}}$, and $u_{0}$ is a positive solution of BVP (1)-(2).

\section{The Existence of Two Positive Solutions}

Theorem 23. Suppose (A2), (A3), and

(A5) $\lambda f^{0, \rho^{\prime}} \leq m$ for some $\rho^{\prime}>0$.

Then (1)-(2) had at least two positive solutions.

Proof. By (A5), we have

$$
\begin{aligned}
T u(t) & =\lambda \int_{0}^{1} G(t, q s) g(s) f(s, u(s)) d_{q} s \\
& \leq \int_{0}^{1} G(t, q s) g(s) \rho^{\prime} m d_{q} s
\end{aligned}
$$

so that $\|T u\| \leq \rho^{\prime}=\|u\|$, for all $u \in \partial V_{\rho^{\prime}}$. Now Lemma 8 yields

$$
i_{k}\left(T, V_{\rho^{\prime}}\right)=1
$$

On the other hand, in view of (A2), we may take $\rho^{*}>\rho^{\prime}$ so that (69) holds (see the proof of Theorem 21). From (A3), we may take $R_{1} \in\left(0, \rho^{\prime}\right)$ so that (77) holds (see the proof of Theorem 22).

Combining (88), (69), and (77), we arrive at

$$
\begin{aligned}
& i_{k}\left(T, K_{\rho^{*}} \backslash \bar{V}_{\rho^{\prime}}\right)=0-1=-1, \\
& i_{k}\left(T, V_{\rho^{\prime}} \backslash \bar{K}_{R_{1}}\right)=1-0=1 .
\end{aligned}
$$

Consequently, $T$ has at least two fixed points, with one on $K_{\rho^{*}} \backslash \bar{V}_{\rho^{\prime}}$ and the other on $V_{\rho^{\prime}} \backslash \bar{K}_{R_{1}}$. Therefore, (1)-(2) had at least two positive solutions.

Theorem 24. Suppose (A1), (A4), and

(A6) $\lambda f_{\rho^{\prime}, \rho^{\prime} / c} \geq M$ for some $\rho^{\prime}>0$.

Then (1)-(2) had at least two positive solutions.

Proof. By (A6), we have

$$
\begin{aligned}
T u(t) & =\lambda \int_{0}^{1} G(t, q s) g(s) f(s, u(s)) d_{q} s \\
& \geq \lambda \int_{a}^{b} G(t, q s) g(s) f(s, u(s)) d_{q} s \\
& \geq \int_{a}^{b} G(t, q s) g(s) M \rho^{\prime} d_{q} s,
\end{aligned}
$$


so that $\|T u\| \geq \rho^{\prime}=\|u\|$, for all $u \in \partial V_{\rho^{\prime}}$, and by Lemma 8 this yields

$$
i_{k}\left(T, V_{\rho^{\prime}}\right)=0 .
$$

On the other hand, in view of (A1), we may take $\rho \in\left(0, \rho^{\prime}\right)$ so that (64) holds (see the proof of Theorem 21). In addition, from (A4), we may take $R>\rho^{\prime}$ so that (85) holds (see the proof of Theorem 22).

Combining (91), (64), and (85), we arrive at

$$
\begin{aligned}
& i_{k}\left(T, K_{R} \backslash \bar{V}_{\rho^{\prime}}\right)=1-0=1, \\
& i_{k}\left(T, V_{\rho^{\prime}} \backslash \bar{K}_{\rho}\right)=0-1=-1 .
\end{aligned}
$$

Hence, $T$ has at least two fixed points, with one on $V_{\rho^{\prime}} \backslash \bar{K}_{\rho}$ and the other on $K_{R} \backslash \bar{V}_{\rho^{\prime}}$. Therefore, (1)-(2) had at least two positive solutions.

We illustrate the applicability of these results with some examples.

Example 25. Consider the problem

$$
\begin{aligned}
D_{0.5}{ }^{(2.5)} u(t)+\lambda(5 t+3)\left(\frac{7 u^{2}+u}{u+1}\right)(2+\cos u) & =0, \\
t \in(0,1), & D_{0.5}^{2} u(0)=0, \\
u(0) & =0, \\
D_{0.5} u(1) & =0 .
\end{aligned}
$$

Here we have $g(t)=5 t+3, f(u)=(2+\cos u)\left(\left(7 u^{2}+u\right) /(u+1)\right)$, and $2<\alpha \leq 3$.

It is readily shown that $f^{0}=f_{0}=3, f^{\infty}=21, f_{\infty}=7$.

Also, $3 u \leq f(u) \leq 21 u$ for $u \geq 0$. By calculation, we find $m=0.19722$, and the smallest $M$ calculated is $M(a, b) \approx$ $M(0.484405,1) \approx 0.74665$. We find $\mu_{1} \approx 0.30366$. Hence, by Theorem 21, there is at least one positive solution if $3 \lambda<$ $\mu_{1}$ and $7 \lambda>\mu_{1}$; that is, there is a positive solution if $\lambda \epsilon$ $(0.47047,1.09773)$.

By Theorem 22, there does not exist a positive solution if either $3 \lambda>\mu_{1}$ or $21 \lambda<\mu_{1}$; that is, if $\lambda<1.09773$ or $\lambda>$ 0.15682 no positive solution exists.

\section{Conflict of Interests}

The authors declare that there is no conflict of interests regarding the publication of this paper.

\section{References}

[1] N. Pongarm, S. Asawasamrit, J. Tariboon, and S. K. Ntouyas, "Multi-strip fractional $q$-integral boundary value problems for nonlinear fractional $q$-difference equations," Advances in Difference Equations, vol. 2014, no. 1, article 193, 2014.
[2] R. Almeida and N. Martins, "Existence results for fractional $q$ difference equations of order $\alpha \in$ ]2, 3[ with three-point boundary conditions," Communications in Nonlinear Science and Numerical Simulation, vol. 19, no. 6, pp. 1675-1685, 2014.

[3] Y. Zhao, H. Chen, and Q. Zhang, "Existence results for fractional $q$-difference equations with nonlocal $q$-integral boundary conditions," Advances in Difference Equations, vol. 2013, article 48, 2013.

[4] R. A. Ferreira, "Positive solutions of a nonlinear $q$-fractional difference equation with integral boundary conditions," International Journal of Difference Equations, vol. 9, no. 2, pp. 135145, 2014.

[5] B. Ahmad and S. K. Ntouyas, "Fractional q-difference hybrid equations and inclusions with Dirichlet boundary conditions," Advances in Difference Equations, vol. 2014, article 199, 2014.

[6] B. Ahmad, S. K. Ntouyas, and A. Alsaedi, "Existence of solutions for fractional $q$-integro-difference inclusions with fractional $q$ integral boundary conditions," Advances in Difference Equations, vol. 2014, article 257, 2014.

[7] R. P. Agarwal, B. Ahmad, A. Alsaedi, and H. Al-Hutami, "On nonlinear fractional $q$-difference equations involving two fractional orders with three-point nonlocal boundary conditions," Dynamics of Continuous, Discrete \& Impulsive Systems Series A: Mathematical Analysis, vol. 21, no. 1, pp. 135-151, 2014.

[8] M. El-Shahed and M. A. Al-Yami, "Positive solutions of boundary value problems for nth order q-differential equations," International Journal of Mathematical Archive, vol. 2, pp. 521$532,2011$.

[9] J. Ma and J. Yang, "Existence of solutions for multi-point boundary value problem of fractional $q$-difference equation," Electronic Journal of Qualitative Theory of Differential Equations, vol. 92, pp. 1-10, 2011.

[10] P. M. Rajković, S. D. Marinković, and M. S. Stanković, "Fractional integrals and derivatives in q-calculus," Applicable Analysis and Discrete Mathematics, vol. 1, no. 1, pp. 311-323, 2007.

[11] Y. Zhao, H. Chen, and B. Qin, "Multiple solutions for a coupled system of nonlinear fractional differential equations via variational methods," Applied Mathematics and Computation, vol. 257, pp. 417-427, 2015.

[12] W.-X. Zhou, X. Liu, and J.-G. Zhang, "Some new existence and uniqueness results of solutions to semilinear impulsive fractional integro-differential equations," Advances in Difference Equations, vol. 2015, article 38, 2015.

[13] Q. Yuan and W. Yang, "Positive solutions of nonlinear boundary value problems for delayed fractional q-difference systems," Advances in Difference Equations, vol. 2014, no. 1, article 51, 16 pages, 2014.

[14] W. Yang, "Positive solutions for nonlinear semipositone fractional $q$-difference system with coupled integral boundary conditions," Applied Mathematics and Computation, vol. 244, pp. 702-725, 2014.

[15] J. R. L. Webb and K. Q. Lan, "Eigenvalue criteria for existence of multiple positive solutions of nonlinear boundary value problems of local and nonlocal type," Topological Methods in Nonlinear Analysis, vol. 27, no. 1, pp. 91-116, 2006.

[16] J. R. L. Webb, "Nonlocal conjugate type boundary value problems of higher order," Nonlinear Analysis: Theory, Methods \& Applications, vol. 71, no. 5-6, pp. 1933-1940, 2009.

[17] J. R. L. Webb and G. Infante, "Non-local boundary value problems of arbitrary order," Journal of the London Mathematical Society, vol. 79, no. 1, pp. 238-258, 2009. 
[18] J. R. Webb and G. Infante, "Positive solutions of nonlocal boundary value problems involving integral conditions," Nonlinear Differential Equations and Applications NoDEA, vol. 15, no. 1-2, pp. 45-67, 2008.

[19] J. R. L. Webb and G. Infante, "Positive solutions of nonlocal boundary value problems: a unified approach," Journal of the London Mathematical Society, vol. 74, no. 3, pp. 673-693, 2006.

[20] V. Kac and P. Cheung, Quantum Calculus, Springer Science \& Business Media, 2002.

[21] R. A. C. Ferreira, "Nontrivial solutions for fractional $q$ difference boundary value problems," Electronic Journal of Qualitative Theory of Differential Equations, no. 70, pp. 1-10, 2010.

[22] M. S. Stanković, P. M. Rajković, and S. D. Marinković, "On $q$ fractional derivatives of Riemann-Liouville and Caputo type," http://arxiv.org/abs/0909.0387.

[23] M. H. Annaby and Z. S. Mansour, q-Fractional Calculus and Equations, vol. 2056 of Lecture Notes in Mathematics, Springer, Berlin, Germany, 2012.

[24] M. El-Shahed and W. M. Shammakh, "Multiple positive solutions for nonlinear fractional eigenvalue problem with non local conditions," Fractional Calculus and Applied Analysis, vol. 3, pp. $1-13,2012$. 


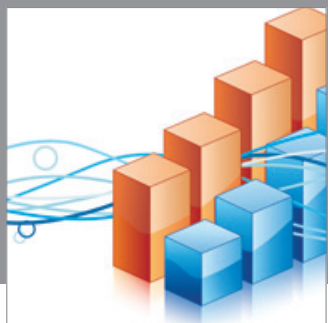

Advances in

Operations Research

mansans

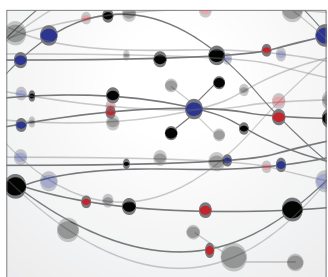

The Scientific World Journal
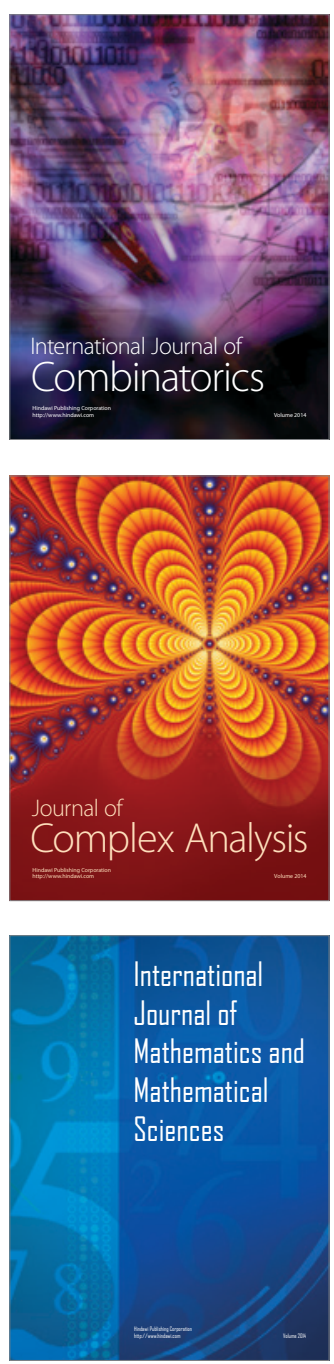
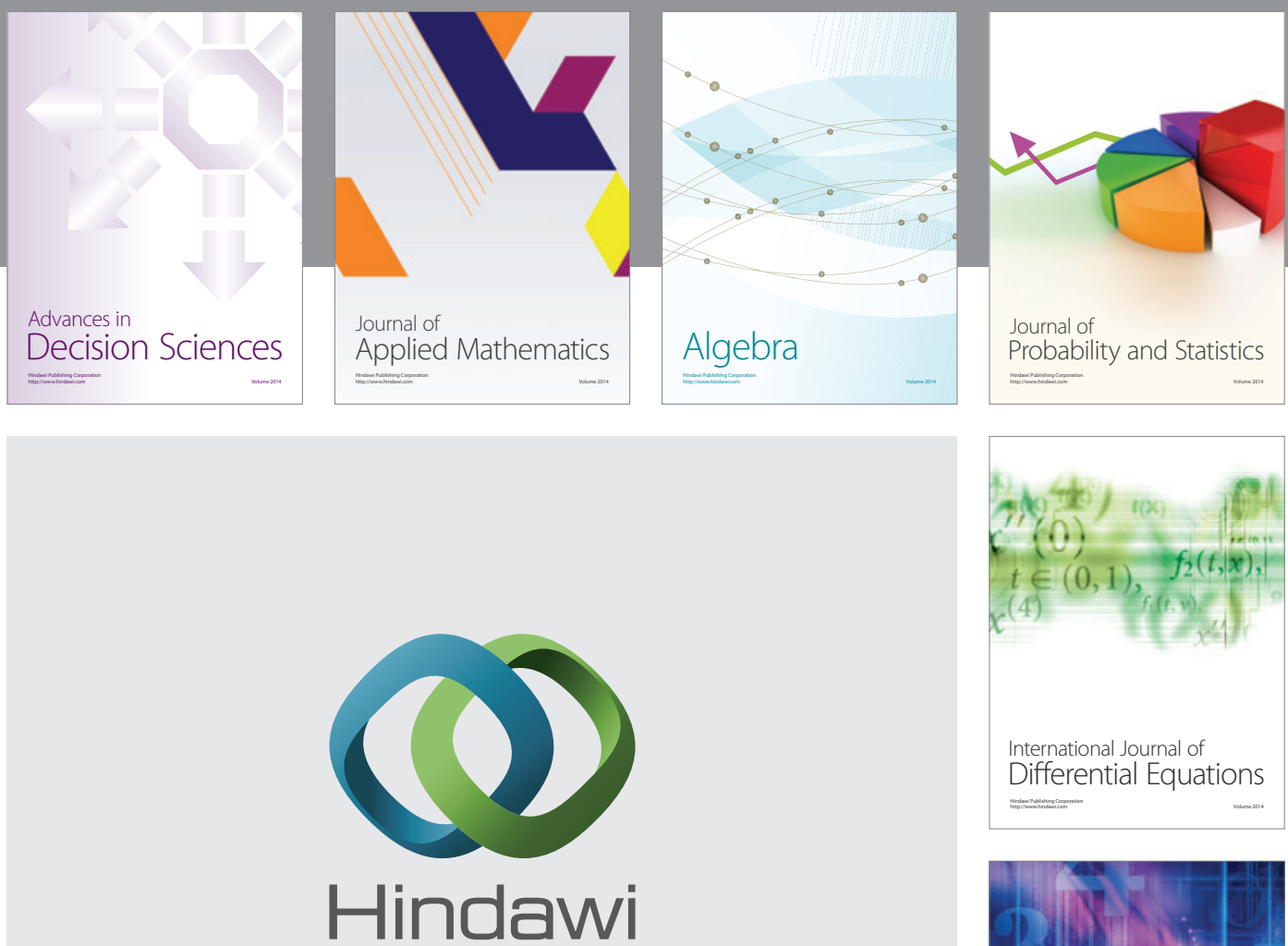

Submit your manuscripts at http://www.hindawi.com
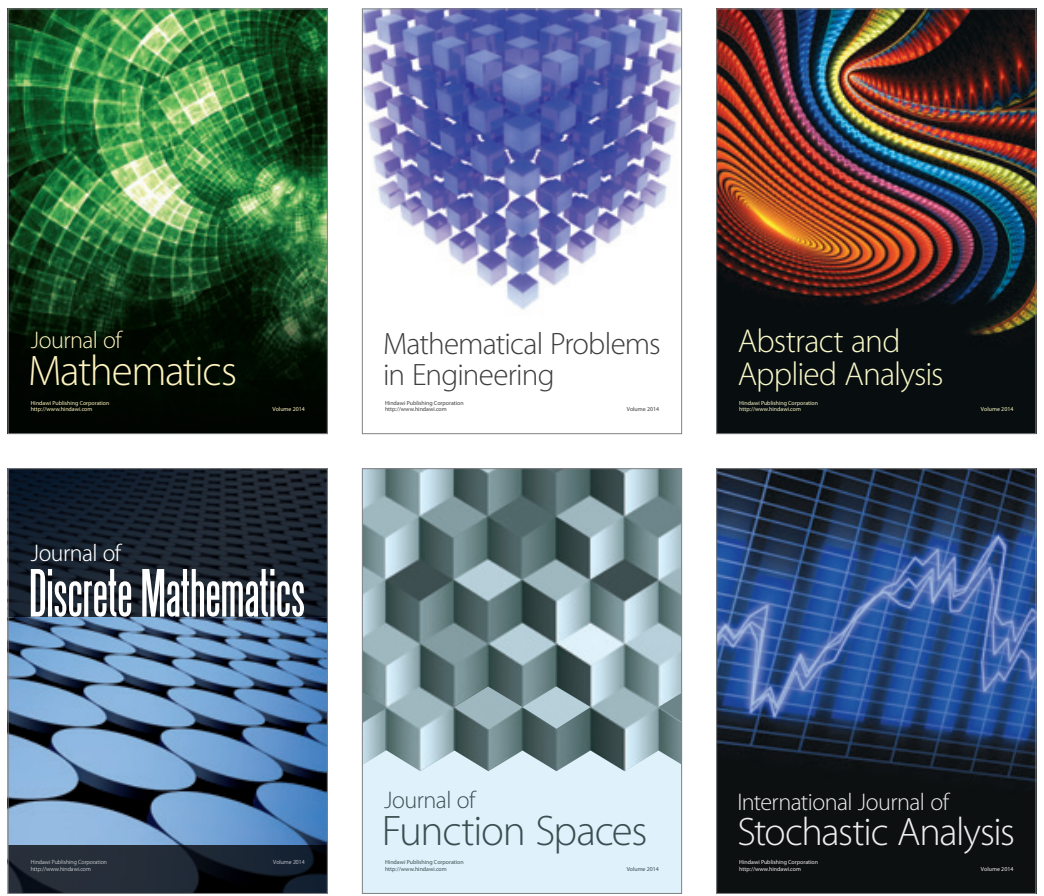

Journal of

Function Spaces

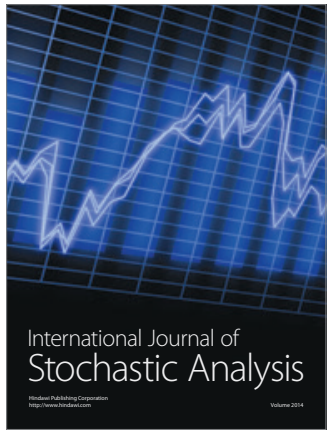

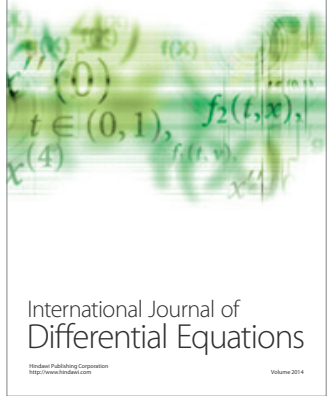
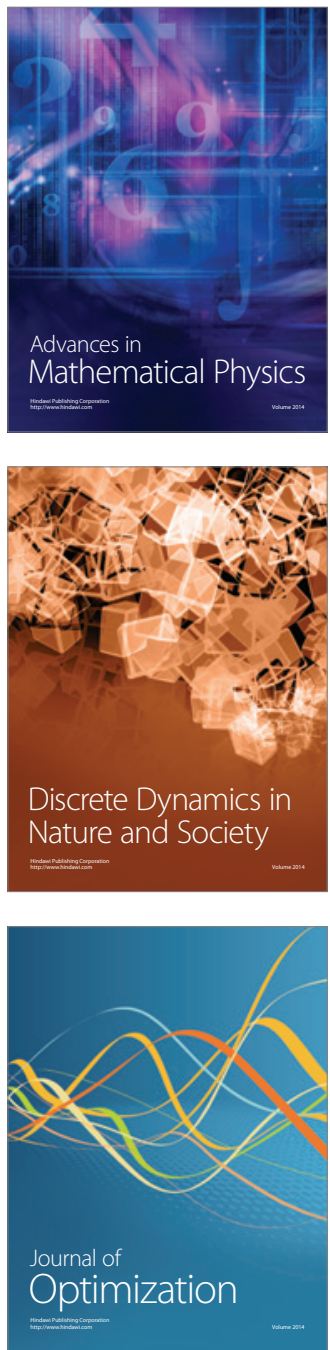